\title{
Binding ability of chitinase onto cellulose: an atomic force microscopy study
}

Polymer Journal (2011) 43, 742-744; doi:10.1038/pj.2011.60

\section{INTRODUCTION}

Polysaccharides have attracted considerable attention because of their potential use in the production of environmentally friendly materials, bio-fuels and other applications. ${ }^{1-3}$ Cellulose $(\beta-1,4$-linked glucose) and chitin $(\beta$-1,4-linked $N$-acetylglucosamine) are, respectively, the first and second most abundant natural polymers, and are degraded by cellulase and chitinase, respectively. Among polysaccharide-degrading enzymes with binding modules, the initial step in the hydrolysis of polymer chains is the binding of the enzyme to the substrate. ${ }^{4,5}$ Therefore, the binding event of the enzyme onto the polysaccharide surface was studied in the present investigation.

Atomic force microscopy (AFM) is a powerful tool for observing surface topographies and measuring interaction forces (also known as AFM force spectroscopy). ${ }^{6-8}$ In our previous work, ${ }^{9}$ we applied force spectroscopy to measure the interaction force between chitin and two different kinds of chitin-binding domains (ChBDs) of a multidomain chitinase. The chitinase is produced by Thermococcus kodakarensis KOD1, a hyperthermophilic archaeon, and is composed of five characteristic domains, including dual catalytic domains (CatD A and B) and triple ChBDs (ChBD1, ChBD2 and ChBD3). ${ }^{10,11}$ All ChBDs can bind to the substrate surface; however, they have different roles that have been proposed on the basis of their different binding forces. For instance, the binding force of ChBD2 is 1.5 times stronger than that of ChBD1. The purpose of ChBD2 and ChBD3, which have nearly identical amino-acid sequences (85\% identical within 100 amino-acid residues), is to secure the initial footing onto the chitin surface. The strong binding of ChBD2 and ChBD3 promotes endo-type cleavage of the chitin chain by CatD B. Subsequently, the chain ends are transported by ChBD1 to exotype CatD A. ${ }^{9}$

To further elucidate the substrate specificity and binding mechanism of ChBD, we investigated the effect of different substrates on the binding force of ChBD. Cellulose, which is structurally different from chitin due to the side chain group, was used as a substrate, as shown in Scheme 1. Owing to its role in the initial binding event of the degradation process, ChBD2 was selected for the present investigation. To the best of our knowledge, the binding force of chitinase onto cellulose has not been previously measured by AFM.

\section{EXPERIMENTAL PROCEDURE}

All of the chemicals employed in the present study were used without further purification. Oriented cellulose films from Cladophora sp., a green alga, were prepared according to a previously published method. ${ }^{12}$ The film was fixed with a water-resistant glue on a silicon support $\left(1 \times 1 \mathrm{~cm}^{2}\right)$, which was cleaned with an ultraviolet-ozone cleaner. Purification of recombinant ChBD2 from Escherichia coli cells and functionalization of the AFM cantilever tip were performed according to a previously reported method. ${ }^{9}$ Briefly, a gold-coated AFM cantilever (OMCL-TR400PB-1, Olympus, Tokyo, Japan) was washed with ultravioletozone cleaner and was functionalized using $\mathrm{HS}\left(\mathrm{CH}_{2}\right)_{11}\left(\mathrm{OCH}_{2} \mathrm{CH}_{2}\right)_{6} \mathrm{OCH}_{2} \mathrm{COOH}\left(\mathrm{PEG}_{6}-\right.$ $\mathrm{COOH}$; Prochimia, Gdansk, Poland). Subsequently, the carboxylic acid groups were connected

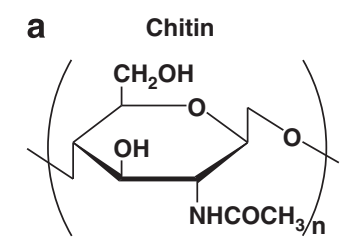

Scheme 1 Chemical structure of chitin (a) and cellulose (b)

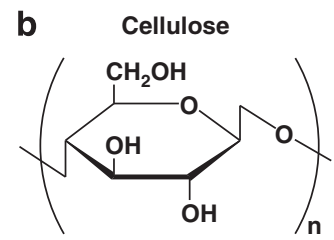

with $\mathrm{N}$-(5-amino-1-carboxypentyl) iminodiacetic acid (Dojindo, Kumamoto, Japan) through an amide bond. Finally, His-tagged ChBD2 was immobilized onto the AFM tip via coordination bonds to $\mathrm{Ni}$ (II) ions.

The surface morphology of cellulose on a silicon support was observed by optical microscopy (Olympus BXFM-F) and AFM (SPI4000/SPA400; SII Nanotechnology Inc, Chiba, Japan). Cellulose film on a silicon support was immersed into a $0.1 \mathrm{M}$ Tris- $\mathrm{HCl}$ buffer solution ( $\mathrm{pH} 7.5)$, and the surface morphology of cellulose was observed under the tapping mode using a cantilever tip without chemical modifications (OMCLAC240-TSW2, Olympus). The binding force of ChBD onto the surface of cellulose was measured under the force curve mode, and a modified AFM cantilever tip was employed at a loading rate of $2 \mathrm{nN} \mathrm{s}^{-1}$. Over 1000 data points were collected, and different tips and cellulose films were applied. The force data were analyzed using SPIP software (Image Metrology, Hørsholm, Denmark).

\section{RESULTS AND DISCUSSION}

The surface morphology of cellulose on a silicone support was visualized using optical microscopy and AFM. Figure 1 shows optical and AFM images of the cellulose film. The orientation of cellulose was observed in the images, as shown in Figure 1a. AFM revealed the nanostructure of the film, and fibrous structures were detected throughout the material. Most of the microfibrils possessed 

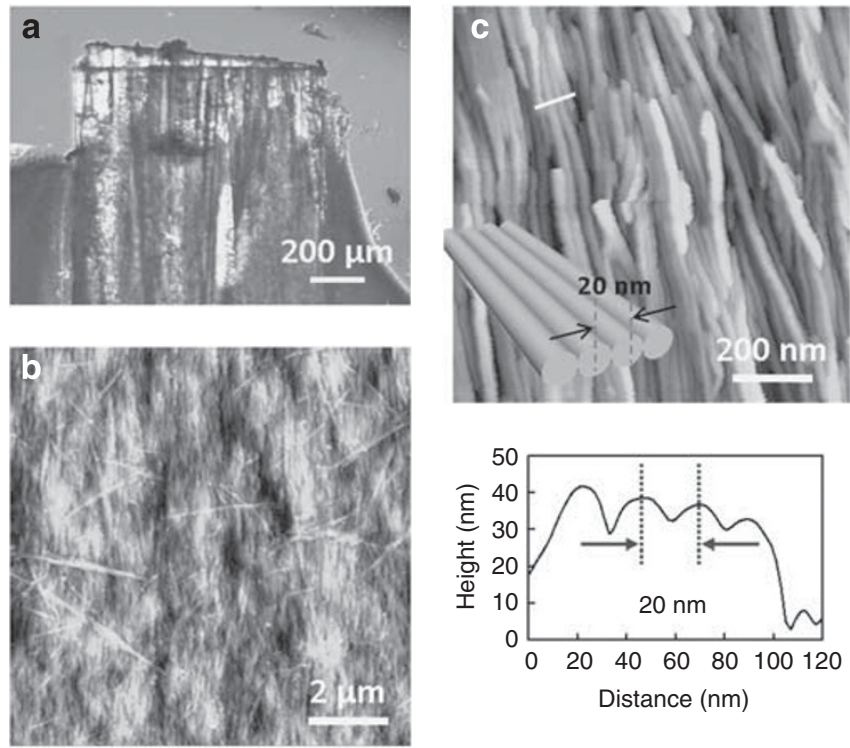

Figure 1 Optical (a) and atomic force microscopy images $(\mathbf{b}, \mathbf{c})$ of the oriented cellulose film on a silicon support. The white-lined region in the frame consists of cross-sectional data (c). The inset within the frame (c) is a schematic representation of cellulose microfibrils with a width of $20 \mathrm{~nm}$. A full color version of this figure is available at Polymer Journal online.
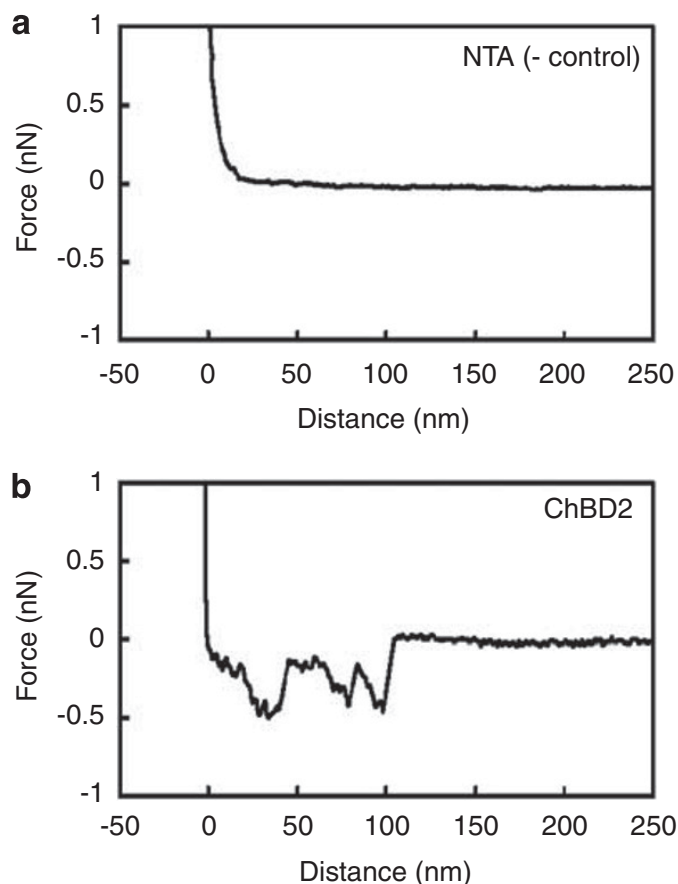

Figure 2 Typical force curve acquired with nitrilotriacetic acid (NTA)-terminated (a) and chitin-binding domain 2 (ChBD2)-functionalized atomic force microscopy tips (b). Only the retraction curve is shown.

a width of $20 \mathrm{~nm}$ and were oriented perpendicular to the direction of the images shown in Figures $1 b$ and $c$.

The binding force of $\mathrm{ChBD} 2$ onto the cellulose surface was measured using AFM in a buffered solution. In the negative control experiment, the force curve was obtained with AFM tips terminated with nitrilotriace- tic acid. Figure 2a shows a typical force curve obtained from the AFM tip without the enzyme. Nonspecific interactions between the tip and the cellulose surface were not detected because of the presence of a hydrophilic ethylene glycol unit in the linker region. ${ }^{13}$ Force curves without interaction peaks were observed at a frequency of $90 \%$.
In contrast, the force curve obtained with a ChBD2-functionalized AFM tip displayed multiple interaction peaks corresponding to the rupture force between ChBD2 and the cellulose surface (Figure 2b). This result indicates that $\mathrm{ChBD} 2$ can bind to the surface of cellulose. Force curves with multiple rupture events were observed at a frequency of $30 \%$.

The single binding force of ChBD2 onto cellulose was analyzed by collecting the force curves. The number of enzymes participating in the binding event cannot be directly counted; thus, histogram analysis was performed. ${ }^{14}$ Figure 3 shows the histogram constructed from ca 500 force curve datasets. Fast Fourier transform smoothing and Gaussian function fitting were performed to determine the local maxima in the data. The difference in the local maxima force (that is, the interval between the red peaks shown in Figure 3) corresponds to the rupture of a single enzyme-substrate bond. The results revealed that the binding force of ChBD2 onto cellulose was $84 \pm 13 \mathrm{pN}$, which was almost identical to that of chitin at a similar loading rate (ca $\left.2 \mathrm{nN} \mathrm{s}^{-1}\right){ }^{9}$

In our previous work, ${ }^{9}$ we determined the binding force of ChBD1 and ChBD2 on the surface of chitin, and the results were compared with the tertiary structures of ChBDs, which were deduced from homology modeling. The binding force of ChBDs is proportional to the number of conserved, surface-exposed, aligned aromatic residues in the enzyme. Carbohydrates with nonpolar surfaces often display stacking interactions with aromatic residues in enzymes. This hydrophobic interaction is strong in aqueous media and is one of the major components of the intermolecular interaction between the substrate and the enzyme. ${ }^{4,5}$

In the present study, the binding force of ChBD2 onto cellulose was comparable to that of chitin, which suggests that the binding ability of ChBD2 to the surface of cellulose and chitin is similar. In both of these systems, carbohydrate-aromatic interactions are the driving force for binding, and the side chains on cellulose do not affect the binding ability of ChBD. The frequency of binding events of ChBD2 onto cellulose (30\%) was slightly lower than that of chitin (39\%). ${ }^{9}$ Currently, the origin of these differences and their effect on the binding of the enzyme to the polysaccharide surface remains unclear. To determine the mechanism of binding, the binding force of the enzyme on the polysaccharide surface must be further analyzed; thus, the energy landscape of enzymatic binding is under investigation. 


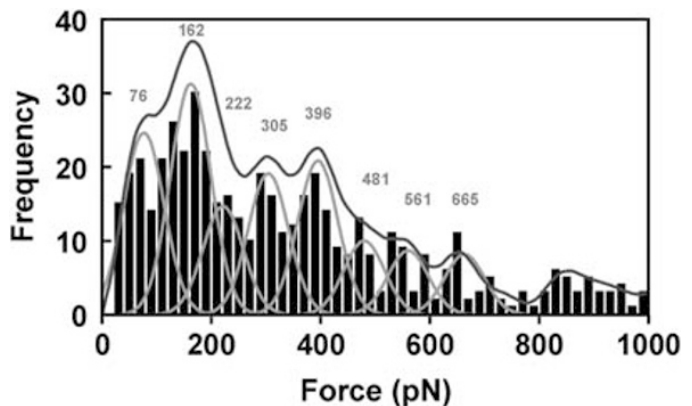

Figure 3 Histogram of pull-off forces for the binding of chitin-binding domain 2 onto cellulose. The blue and red curves correspond to fast Fourier transform-smoothed and Gaussian fitted data, respectively. The force values for each peak are indicated in the histogram. A full color version of this figure is available at Polymer Journal online.

\section{CONCLUSION}

Force curve measurements of the binding of ChBD2 on the surface of cellulose were obtained, and the data were compared with that of chitin. Preliminary results revealed that the binding forces of ChBD2 onto cellulose and chitin are nearly equivalent, suggesting that the side chains on the polysaccharide have a limited effect on the binding of ChBD2. Thus, the binding of ChBD onto cellulose/chitin can be attributed to interactions between aromatic residues in the enzyme and the pyranose ring of the polysaccharide.

\section{ACKNOWLEDGEMENTS}

This work was partly supported by the Mitsubishi Chemical Corporation Fund and MEXT KAKENHI (21106522). We thank Dr Seiji Tsuzuki of the Nanosystem Research Institute, National Institute of Advanced Industrial Science and Technology, for helpful discussions on the interactions between carbohydrates and ChBDs.
Yoshihiro Kikkawa ${ }^{1}$, Masato Fukuda ${ }^{2}$, Ayumi Kashiwada $^{2}$, Kiyomi Matsuda ${ }^{2}$, Masatoshi Kanesato ${ }^{1}$, Masahisa Wada ${ }^{3,4}$, Tadayuki Imanaka ${ }^{5}$ and Takeshi Tanaka ${ }^{1}$

${ }^{1}$ National Institute of Advanced Industrial Science and Technology (AIST), Tsukuba Central 4, Ibaraki, Japan; ${ }^{2}$ Department of Applied Molecular Chemistry, College of Industrial Technology, Nihon University, Chiba, Japan; ${ }^{3}$ Department of Biomaterials Science, Graduate School of Agricultural and Life Sciences, The University of Tokyo,

Tokyo, Japan; ${ }^{4}$ Department of Plant and Environmental New Resources, College of Life Sciences, Kyung Hee University, Gyeonggi-do, Republic of Korea and ${ }^{5}$ Department of Biotechnology, College of Life Sciences, Ritsumeikan University, Shiga, Japan E-mail: y.kikkawa@aist.go.jp or tanaka-t@aist.go.jp
1 Prashanth, K. V. H. \& Tharanathan, R. N. Chitin/Chitosan: modifications and their unlimited application potentialan overview. Trends Food Sci. Tech. 18, 117-131 (2007).

2 Wegner, T. H. \& Jones, E. P. in The Nanoscience and Technology of Renewable Biomaterials (eds Lucia, L. A. \& Rojas, O. J.) Ch. 1, 1-41 (John Wiley \& Sons Ltd., West Sussex, UK, 2009).

3 Alvira, P., Tomás-Pejó, E., Ballesteros, E. M. \& Negro, M. J. Pretreatment technologies for an efficient bioethanol production process based on enzymatic hydrolysis: a review. Biores. Tech. 101, 4851-4861 (2010).

4 Boraston, A. B., Bolam, D. N., Gilbert, H. J. \& Davies, G. J. Carbohydrate-binding modules: fine-tuning polysaccharide recognition. Biochem. J. 382, 769-781 (2004).

5 Shoseyov, O., Shani, Z. \& Levy, I. Carbohydrate binding modules: biochemical properties and novel applications. Microbiol. Mol. Biol. Rev. 70, 283-295 (2006).

6 Hugel, T. \& Seiz, M. The study of molecular interactions by AFM force spectroscopy. Macromol. Rapid Commun. 22, 989-1016 (2001)

7 Zou, S., Schönherr, H. \& Vancso, G. J. in Scanning Probe Microscopies Beyond Imaging (ed. Samorì, P.) Ch. 11, 315-353 (Wiley-VCH Verlag GmbH \& Co., KGaA, Weinheim, Germany, 2006).

8 Bizzarri, A. R. \& Cannistraro, S. Atomic force spectroscopy in biological complex formation: strategies and perspectives. J. Phys. Chem. B 113, 16449-16464 (2009).

9 Kikkawa, Y., Tokuhisa, H., Shingai, H., Hiraishi, T., Houjou, H., Kanesato, M., Imanaka, T. \& Tanaka, T. Interaction force of chitin-binding domains onto chitin surface. Biomacromolecules 9, 2126-2131 (2008).

10 Tanaka, T., Fujiwara, S., Nishikori, S., Fukui, T., Takagi, M. \& Imanaka, T. A unique chitinase with dual active sites and triple substrate binding sites from hyperthermophilic archaeon Pyrococcus kodakaraensis KOD1. Appl. Environ. Microbiol. 65, 5338-5344 (1999).

11 Tanaka, T., Fukui, T. \& Imanaka, T. Different cleavage specificities of the dual catalytic domains in chitinase from the hyperthermophilic archaeon Thermococcus kodakaraensis KOD1. J. Biol. Chem. 276, 3562935635 (2001).

12 Nishiyama, Y., Kuga, S., Wada, M. \& Okano, T. Cellulose microcrystal film of high uniaxial orientation. Macromolecules 30, 6395-6397 (1997).

13 Sigal, G. B., Bamdad, C., Barberis, A., Strominger, J. \& Whitesides, G. M. A self-assembled monolayer for the binding and study of histidine-tagged proteins by surface plasmon resonance. Anal. Chem. 68, 490-497 (1996).

14 Zapotoczny, S., Auletta, T., de Jong, M. R., Schönherr, H., Huskens, J., van Veggel, F., Reinhoudt, D. N. \& Vancso, G. J. Chain length and concentration dependence of $\beta$-cyclodextrin-ferrocene host-guest complex rupture forces probed by dynamic force spectroscopy. Langmuir 18, 6988-6994 (2002). 